\title{
Evaluation of the One Loop String Path Integral
}

\author{
Joseph Polchinski
}

Theory Group, Physics Department, University of Texas, Austin, TX 78712 USA

\begin{abstract}
We evaluate Polyakov's path integral for the sum over all closed surfaces with the topology of a torus, in the critical dimension $d=26$. The result is applied to the partition function and cosmological constant of the free bosonic string, and to tachyon scattering amplitudes.
\end{abstract}

In this paper, we evaluate the sum over all closed 2-surfaces with the topology of a torus. Our starting point is the path integral of Polyakov [1]:

$$
W=\int \frac{d g_{a b} d x^{\mu}}{V_{G C} V_{W}} \exp \left(-\int d^{2} \sigma \sqrt{g}\left[\frac{T}{2} g^{a b} \partial_{a} x^{\mu} \partial_{b} x^{\mu}+\lambda R+\mu^{2}\right]\right) .
$$

The integration runs over all Euclidean metrics $g_{a b}(\sigma)$ on a two-surface of given topology, and all embeddings $x^{\mu}(\sigma)$ of the 2-surface into $d$-dimensional Euclidean spacetime. $T$ is the string tension. The $\lambda R$ term is proportional to the Euler number of the surface, and vanishes for the torus. The action is invariant under changes of the coordinates $\sigma$ of the world sheet. Classically, when $\mu^{2}=0$, there is a second local symmetry group, the Weyl transformations

$$
\delta g_{a b}(\sigma)=\lambda(\sigma) g_{a b}(\sigma) .
$$

This remains a symmetry of the quantum theory provided $d=26$ and provided the counterterm $\mu^{2}$ is appropriately chosen. We restrict our attention to this case, of exact Weyl invariance. The volumes of the local symmetry groups, $V_{G C}$ and $V_{W}$ respectively, must be divided out of the integration. We show that this can be done in an unambiguous way, leaving a finite measure; genus 1 , the torus, is particularly simple in this respect. Our result is not new, since the one loop closed string graph has been evaluated by operator methods [2]. However, it is useful to obtain it directly from (1). Friedan [3] and Alvarez [4] have given general discussions of the sums over surfaces with handles. As a check, we calculate the free energy of a gas of free strings, and also compare with the expression for one loop string graphs as obtained by operator methods [2]. For the one loop cosmological constant of the free bosonic string theory we find a surprising result: it is not equal to the sum of the one loop contributions of the individual particles.

In order to describe a torus, we take $x^{\mu}$ and $g_{a b}$ to be periodic functions of $\sigma^{1}$ and 
$\sigma^{2}$

$$
\begin{gathered}
x^{\mu}\left(\sigma^{1}+1, \sigma^{2}\right)=x^{\mu}\left(\sigma^{1}, \sigma^{2}+1\right)=x^{\mu}\left(\sigma^{1}, \sigma^{2}\right), \\
g_{a b}\left(\sigma^{1}+1, \sigma^{2}\right)=g_{a b}\left(\sigma^{1}, \sigma^{2}+1\right)=g_{a b}\left(\sigma^{1}, \sigma^{2}\right) .
\end{gathered}
$$

Thus the unit cell is simply $0<\sigma^{1}<1,0<\sigma^{2}<1$. Any metric can be brought by a general coordinate transformation (respecting the periodicity (3)) to the form [5]

$$
d s^{2}=g_{a b} d \sigma^{a} d \sigma^{b}=e^{\phi(\sigma)}\left|d \sigma^{1}+\tau d \sigma^{2}\right|^{2}
$$

with $\tau$ a complex number, $\operatorname{Im}(\tau)>0$, referred to as a modular parameter. The metric (4) is equivalent to a metric $g_{a b} \alpha \delta_{a b}$ on a parallelogram whose sides are defined by the complex numbers 1 and $\tau$. It is more convenient, however, to keep the coordinate region fixed on the unit square and let $\tau$ appear in the metrix. Any small variation of the metric can be resolved into a Weyl transformation plus a general coordinate transformation plus a change in $\tau$ :

$$
\delta g_{a b}(\sigma)=g_{a b}(\sigma) \delta \phi(\sigma)+\delta \xi_{a ; b}(\sigma)+\delta \xi_{b ; a}(\sigma)+g_{a b, i}(\sigma) \delta \tau_{i}
$$

where $\tau_{1}, \tau_{2} \equiv \operatorname{Re}(\tau), \operatorname{Im}(\tau)$. The integral over metrics thus separates into an integral over the Weyl group, an integral over the general coordinate group, and an integral over $\tau$. We wish to determine the Jacobian defined by

$$
d g=(d \phi d \xi)^{\prime} d^{2} \tau J(\phi, \tau) .
$$

The prime denotes the following: the variations

$$
\delta \xi^{a}(\sigma)=\varepsilon^{a}, \quad \delta \phi(\sigma)=-\varepsilon^{a} \partial_{a} \phi(\sigma)
$$

for constant $\varepsilon^{a}$ give $\delta g_{a b}=0$. The prime restricts the variations of $\xi$ and $\phi$ to be orthogonal to the zero mode (7). This reflects the fact that Eq. (4) is not a complete choice of gauge: translations preserve the form (4).

Before defining the measures appearing in (6), let us, following [1], define metrics for small variations in the fields. The natural metric for small $\delta g_{a b}$ is

$$
\|\delta g\|^{2}=\int d^{2} \sigma \sqrt{g}\left(g^{a c} g^{b d}+C g^{a b} g^{c d}\right) \delta g_{a b} \delta g_{c d}
$$

with $C$ an arbitrary constant. Up to normalization, this is the most general invariant without derivatives. Similarly, define

$$
\begin{aligned}
\|\delta \phi\|^{2} & =\int d^{2} \sigma \sqrt{g} \delta \phi^{2}, \\
\|\delta \xi\|^{2} & =\int d^{2} \sigma \sqrt{g} g^{a b} \delta \xi_{a} \delta \xi_{b}, \\
\|\delta x\|^{2} & =\int d^{2} \sigma \sqrt{g} \delta x^{\mu} \delta x^{\mu} .
\end{aligned}
$$

For a finite dimensional space, the metric would define a measure, but we cannot here define a measure explicitly. Instead, we define it implicitly, in terms of the values of Gaussian integrals:

$$
\begin{aligned}
\int d \delta g e^{-\|\delta\|^{2} / 2} & =1 \\
\int d \delta \phi e^{-\|\delta \phi\|^{2} / 2} & =1
\end{aligned}
$$




$$
\begin{aligned}
& \int d \delta \xi e^{-\|\delta \xi\|^{2} / 2}=1, \\
& \int d \delta x e^{-\|\delta x\|^{2} / 2}=1
\end{aligned}
$$

Later, we will discuss the extent to which $W$ depends on our various definitions. In (9a), $\delta g$ is not a finite change in the metric, but rather a finite element of the tangent space to the space of metrics, at a given metric $g_{a b}$. Thus, (9a) defines a measure on the tangent space; this can be identified with the measure of interest, $d g .{ }^{1}$ The same applies to ( $9 \mathrm{~b}-\mathrm{d})$. For completeness, we also list

$$
\int d^{2} \delta \tau e^{-\delta \tau_{i} \delta \tau_{i} / d^{2} \sigma \sqrt{ } g / 2}=\frac{2 \pi}{\int d^{2} \sigma \sqrt{g}}
$$

where $d^{2} \delta \tau$ is simply $d \delta \tau_{1} d \delta \tau_{2}$.

The integration $d \phi d \xi$ can be separated into

$$
d \phi d \xi=(d \phi d \xi)^{\prime} d \varepsilon^{1} d \varepsilon^{2} .
$$

The integrals defining $d \phi$ and $d \xi$ also separate:

$$
\begin{aligned}
1 & =\int d \varepsilon^{1} d \varepsilon^{2} e^{-Q_{a b} \varepsilon^{a} \varepsilon^{b} / 2} \int(d \delta \phi d \delta \xi)^{\prime} e^{-\left(\|\delta \phi\|^{2}+\|\delta \xi\|\right)^{2} / 2} \\
& =2 \pi\left(\operatorname{det}^{-1 / 2} Q_{a b}\right) \int(d \delta \phi d \delta \xi)^{\prime} e^{-\left(\|\delta \phi\|^{2}+\|\delta \xi\|\right)^{2} / 2},
\end{aligned}
$$

where $Q_{a b}=\int d^{2} \sigma \sqrt{g}\left(\partial_{a} \phi \partial_{b} \phi+g_{a b}\right)$. This gives the normalization of $(d \phi d \xi)^{\prime}$. To obtain the Jacobian $J(\phi, \tau)$, rewrite the integral defining $d \delta g$ in terms of $\delta \phi, \delta \xi$, and $\delta \tau$ :

$$
1=J(\phi, \tau) \int(d \delta \phi d \delta \xi)^{\prime} d^{2} \delta \tau \exp -\|\delta g\|^{2} / 2
$$

with

$$
\|\delta g\|^{2}=\left(\delta \phi \delta \xi^{a} \delta \tau_{i}\right) \mathscr{M}\left[\begin{array}{l}
\delta \phi \\
\delta \xi \\
\delta \tau_{j}
\end{array}\right] .
$$

The matrix $\mathscr{M}$ is conveniently written as a product

$$
\begin{aligned}
\mathscr{M}= & {\left[\begin{array}{ccc}
1 & 0 & 0 \\
-D_{a} & \delta_{a}{ }^{c} & 0 \\
\frac{1}{2} g^{e f} g_{e f, i} & 0 & \delta_{i k}
\end{array}\right] } \\
& \times\left[\begin{array}{ccc}
2+4 C & 0 & 0 \\
0 & 2 \Delta_{c}^{d} & -2 D_{e} \chi_{l c}^{e} c \\
0 & 2 \chi_{k}^{e d} D_{e} & \chi_{k e f} \chi_{l}^{e f}
\end{array}\right] \times\left[\begin{array}{ccc}
1 & D^{b} & \frac{1}{2} g^{e f} g_{e f, j} \\
0 & \delta_{d}^{b} & 0 \\
0 & 0 & \delta_{l j}
\end{array}\right] \\
& \equiv \mathscr{T} \mathscr{N} \mathscr{J}^{T} .
\end{aligned}
$$

In (13), $\Delta_{c}{ }^{d}=-\delta_{c}{ }^{d} D^{2}-D^{d} D_{c}+D_{c} D^{d}$ and $\chi_{i a b}=g_{a b, i}-\frac{1}{2} g_{a b} g^{c d} g_{c d, i}$. Note that

1 P. Nelson suggests that this identification can be made precise with a construction based on geodesic normal coordinates 
$\operatorname{det}(\mathscr{T})=1$. From (11) and the normalization (9), (10) one has

$$
J(\phi, \tau)=\frac{\int d^{2} \sigma \sqrt{g}}{\left(\operatorname{det} Q_{a b}\right)^{1 / 2}}\left(\operatorname{det}^{\prime} \mathscr{N}\right)^{1 / 2} .
$$

The upper $2 \times 2$ block of $\mathscr{N}$ is precisely as in Polyakov [1].

To carry out the $x^{\mu}$ integration, separate the constant pieces

$$
x^{\mu}(\sigma)=\bar{x}^{\mu}+x^{\mu^{\prime}}(\sigma),
$$

where $x^{\mu \prime}(\sigma)$ is orthogonal to the constant; $d x=d \bar{x} d x^{\prime}$. In the same fashion as (10), one finds

$$
\int d \delta x^{\mu^{\prime}} e^{-\|\delta x\|^{2} / 2}=\left(\frac{\int d^{2} \sigma \sqrt{g}}{2 \pi}\right)^{1 / 2} .
$$

The integral over $\bar{x}^{\mu}$ diverges and can be regulated by putting the system in a periodic box of dimensions $L^{1} L^{2} \ldots L^{d}$. Then

$$
\int d x e^{-T / 2 \int d^{2} \sigma \sqrt{g} g g^{a b} \partial_{a} x^{\mu} \partial_{b} x^{\mu}}=\left(\prod_{\mu} L^{\mu}\right)\left\{\frac{2 \pi}{\int d^{2} \sigma \sqrt{g}} \operatorname{det}^{\prime}\left(-T \sqrt{g}-1 \partial_{a} g^{a b} \sqrt{g \partial_{b}}\right)\right\}^{-d / 2} .
$$

The sum over surfaces now takes the form

$$
\begin{aligned}
W_{\text {torus }}= & \int \frac{(d \xi d \phi)^{\prime} d^{2} \tau}{V_{G C} V_{W}} \prod_{\mu} L^{\mu}\left\{\left(\operatorname{det}^{\prime} \mathcal{N}\right)^{1 / 2}\left(\int d^{2} \sigma \sqrt{g}\right)^{1+d / 2}\left(\operatorname{det} Q_{a b}\right)^{-1 / 2}(2 \pi)^{-d / 2}\right. \\
& \left.\cdot \operatorname{det}^{\prime}\left(-T \sqrt{g}{ }^{-1} \partial_{a} g^{a b} \sqrt{g} \partial_{b}\right)^{-d / 2}\right\} .
\end{aligned}
$$

The volume of Weyl group is just $\int d \phi$. The general coordinate group is the connected component, $\int d \xi$, times a group $D$ of disconnected transformations which leave the boundary conditions (3) unchanged. A choice of gauge in general fixes some of the discrete transformations and is invariant under a subgroup $\widetilde{D}$. The Fadeev-Popov determinant contains a factor of $\operatorname{order}(D) / \operatorname{order}(\tilde{D})$. The full $V_{G C}$ contains a factor of $\operatorname{order}(D)$. Thus, the final denominator contains a factor of $\operatorname{order}(\widetilde{D})$ :

$$
V_{G C} V_{W} \rightarrow \operatorname{order}(\tilde{D}) \int d \xi \int d \phi .
$$

Two steps are needed before the $\xi$ and $\phi$ integrations can be cancelled between the numerator and denominator. First, the volume of the full and restricted integrations are related:

$$
\int d \phi d \xi=\int(d \phi d \xi)^{\prime} \int_{0}^{1} d \varepsilon^{1} \int_{0}^{1} d \varepsilon^{2}=\int(d \phi d \xi)^{\prime}
$$

since the range of $\varepsilon^{1}$ and $\varepsilon^{2}$ is 0 to 1 . Secondly, the factor \{\} in (18) must be independent of $\phi$. Any dependence comes about from the conformal anomaly. This is a local property and so the dependence is the same as calculated by Polyakov [1], regardless of topology:

$$
\frac{\delta}{\delta \phi} \ln \{\quad\}=\frac{26-d}{96 \pi} \partial^{2} \phi+\left(\Lambda^{2}-\mu^{2}\right) e^{\phi} .
$$


The first term in the integral cannot be cancelled by a local, coordinate invariant counterterm, but it vanishes in the critical dimension $d=26$. In the second term, the sum of all quantum contributions $\Lambda^{2}$ can be cancelled by appropriate choice of $\mu^{2}$. This is natural in the critical dimension, since the Weyl group is then an exact symmetry. In this case, we can now eliminate the $\phi$ and $\xi$ integrations, and also set $\phi(\sigma)=0$ in evaluating (18). Then

$$
\begin{aligned}
& \int d^{2} \sigma \sqrt{g}=\tau_{2}, \quad \operatorname{det} Q_{a b}=\tau_{2}{ }^{4}, \text { and } \\
& \left(\operatorname{det}^{\prime} \mathscr{N}\right)^{1 / 2}=(\operatorname{det}[2+4 C])^{1 / 2}\left(\operatorname{det}^{\prime}\left[-2 \delta_{c}{ }^{d} g^{a b} \partial_{a} \partial_{b}\right]\right)^{1 / 2} \frac{2}{\tau_{2}^{2}}, \\
& \left(\operatorname{det}^{\prime}\left[-2 \delta_{c}{ }^{d} g^{a b} \partial_{a} \partial_{b}\right]\right)^{1 / 2}=\operatorname{det}^{\prime}\left[-2 g^{a b} \partial_{a} \partial_{b}\right] \\
& =\operatorname{det}^{\prime}(2) \operatorname{det}^{\prime}\left(-g^{a b} \partial_{a} \partial_{b}\right) \\
& =\frac{1}{2} \operatorname{det}(2) \operatorname{det}^{\prime}\left(-g^{a b} \partial_{a} \partial_{b}\right) \text {, } \\
& \operatorname{det}^{\prime}\left(-T \sqrt{g}-1 \partial_{a} g^{a b} \sqrt{g} \partial_{b}\right)=\operatorname{det}^{\prime}\left(-g^{a b} \partial_{a} \partial_{b}\right) \operatorname{det}(T) / T .
\end{aligned}
$$

The determinant of $-g^{a b} \partial_{a} \partial_{b}$ requires one counterterm, of the form $\mu^{2}$. The value of this counterterm is completely fixed by Weyl invariance. The determinant is evaluated in the Appendix:

$$
\operatorname{det}^{\prime}\left(-g^{a b} \partial_{a} \partial_{b}\right)=\prod_{n_{1}, n_{2}}^{\prime}\left(4 \pi^{2} g^{a b} n_{a} n_{b}\right)=\tau_{2}{ }^{2} e^{-\pi \tau_{2} / 3}\left|f\left(e^{2 \pi i \tau}\right)\right|^{4}
$$

where

$$
f\left(e^{2 \pi i \tau}\right)=\prod_{n=1}^{\infty}\left(1-e^{2 \pi i n \tau}\right)
$$

As will be discussed below Eq. (28), the determinant of a constant is just a contribution to $\mu^{2}$ and can be neglected.

The diffeomorphisms which respect the orientation, the periodicity (3) and the gauge condition (4) take the form

$$
\sigma_{1} \rightarrow \alpha \sigma_{1}+\beta \sigma_{2}, \quad \sigma_{2} \rightarrow \gamma \sigma_{1}+\delta \sigma_{2},
$$

with $\alpha, \beta, \gamma, \delta$ integers and $\alpha \delta-\beta \gamma=1$. Under (24), the parameter $\tau$ undergoes a modular transformation [2]

$$
\tau \rightarrow \frac{\tau \delta+\beta}{\tau \gamma+\alpha} .
$$

Thus, one can fix most of the invariances (24) by requiring $\tau$ to remain in a fundamental region $F$, e.g.

$$
-\frac{1}{2}<\operatorname{Re} \tau<\frac{1}{2}, \quad \operatorname{Im} \tau>0, \quad|\tau|>1,
$$

Every $\tau$ in the complex upper half plane (except a set of measure zero) can be obtained uniquely as a modular transformation acting on some $\tau \in F$. There remains only a 2 -fold gauge invariance,

$$
\sigma_{1} \rightarrow-\sigma_{1}, \quad \sigma_{2} \rightarrow-\sigma_{2},
$$


so that $\operatorname{order}(\widetilde{D})=2$. Our final expression for the sum over surfaces is

$$
W_{\text {torus }}=T^{13}\left(\prod_{\mu} L^{\mu}\right) \int_{F} \frac{d^{2} \tau}{4 \pi \tau_{2}^{2}} e^{4 \pi \tau_{2}}\left(2 \pi \tau_{2}\right)^{-12}\left|f\left(e^{2 \pi i \tau}\right)\right|^{-48} .
$$

The case we are studying, one loop and no external fields $\left(L=1, N_{e}=0\right)$, is particularly simple in that the overall normalization contains no free parameters. The sum depends on the curvature scalar term in the action (1) as $\exp [8 \pi \lambda(L-1)]$, and so is $\lambda$-independent only at $L=1$. For $N_{e} \neq 0$, one has constants from the normalization of the vertex operators. A related point is the analysis of Weinberg [6], which shows that there is an arbitrary constant $g$ which cannot be determined from unitary and which enters as $g^{-2+2 L+N_{e}}: L=1, N_{e}=0$ is independent of $g$. In fact, the sum over graphs with $L=1, N_{e}=0$ will be related, below, to the free energy of a string gas; thus its normalization is fixed. It should therefore be independent of our various definitions. The sum $W_{\text {torus }}$ is proportional to the measures $d g$ and $d x$ and inversely proportional to $d \xi$ and $d \phi$, defined in (9). The integration (9a) over all metrics breaks up into a product of a separate integration at each coordinate point,

$$
\int d \delta g e^{-\|\delta g\|^{2} / 2}=\prod_{\sigma} \int d \delta g_{\sigma} e^{-\left\|\delta g_{\sigma}\right\|^{2} / 2}
$$

The only invariant quantity which is such a product (and therefore does not depend on derivatives of the metric) is

$$
\exp \int d^{2} \sigma M^{2} \sqrt{g}
$$

$\left(M^{2} \sim \delta^{2}(0)\right)$. Thus, the integral (28) is equal to 1 , up to an adjustment of $\mu^{2}$. The same is true for the other measures $(9 \mathrm{~b}-\mathrm{d})$. By the same argument, the determinant of a constant is only a contribution to $M^{2}$, and a change in the normalization of the metrics (8) can be absorbed into $\mu^{2}$. So, there is no ambiguity in the result (27). The assumption (28), that the measure is local, is the key input that makes it possible to calculate $W_{\text {torus }}$ with no uncertainty in the normalization or $\tau$-dependence.

It is a nice check to see that the sum over all surfaces (not necessarily connected) in the limit $L^{2}, L^{3}, \ldots, L^{26} \rightarrow \infty, L^{1}=\beta$ fixed, reproduces the thermal partition function for a gas of free strings. ${ }^{2}$ For the free energy $F(\beta)$, this is

$$
F(\beta)=-\left(\prod_{\mu} L^{\mu}\right)^{-1} W_{\text {connected }}
$$

The leading $\beta$-dependence in $F(\beta)$ comes from tori which wind $r$ times around the compact 1-direction. The boundary condition (3a) is then modified to

$$
\begin{aligned}
x^{\mu}\left(\sigma^{1}, \sigma^{2}+1\right) & =x^{\mu}\left(\sigma^{1}, \sigma^{2}\right)+r \beta{\delta^{\mu}}_{1} \\
x^{\mu}\left(\sigma^{1}+1, \sigma^{2}\right) & =x^{\mu}\left(\sigma^{1}, \sigma^{2}\right) \\
g_{a b}\left(\sigma^{1}+1, \sigma^{2}\right) & =g_{a b}\left(\sigma^{1}, \sigma^{2}+1\right)=g_{a b}\left(\sigma^{1}, \sigma^{2}\right) .
\end{aligned}
$$

2 Strictly speaking, the $\tau$ integral and the $L^{\mu} \rightarrow \infty$ limit both diverge because the theory contains a tachyon. We take the $L^{\mu} \rightarrow \infty$ limit formally, dropping surfaces which wind around the $2, \ldots, 26$ directions 
It is convenient to separate $x^{\mu}$ into a periodic piece and a linear piece,

$$
x^{\mu}\left(\sigma^{1}, \sigma^{2}\right)=y^{\mu}\left(\sigma^{1}, \sigma^{2}\right)+r \beta \sigma^{2} \delta_{1}^{\mu} .
$$

One has for the action

$$
S(x, g)=S(y, g)+r^{2} \beta^{2} T / 2 \tau_{2},
$$

and so

$$
F(\beta)=-T^{13} \int_{0}^{\infty} \frac{d \tau_{2}}{2 \pi \tau_{2}^{2}} \int_{-1 / 2}^{1 / 2} d \tau_{1} e^{4 \pi \tau_{2}}\left(2 \pi \tau_{2}\right)^{-12}\left|f\left(e^{2 \pi i \tau}\right)\right|^{-48} \sum_{r=1}^{\infty} e^{-r^{2} \beta^{2} T / 2 \tau_{2}} .
$$

Note that the condition (30) fixes some of the discrete invariances (24); only $\delta=1$, $\gamma=0$ (thus $\alpha=1$ ) survives. This is reflected in (33). The expression (33) can be compared with the free energy as calculated from the known spectrum of the free string. The free energy of a single particle is

$$
F\left(\beta, m^{2}\right)=\frac{1}{\beta} \int \frac{d^{d-1} k}{(2 \pi)^{d-1}} \ln \left(1-e^{-\beta \omega_{k}}\right)=-\int_{0}^{\infty} \frac{d s}{s}(2 \pi s)^{-d / 2} \sum_{r=1}^{\infty} e^{-m^{2} s / 2-r^{2} \beta^{2} / 2 s},
$$

where the latter, "proper time," form is convenient for summing on $m^{2}$. In terms of the occupation numbers of the transverse oscillators, $N_{n i}, \tilde{N}_{n i}$, the spectrum is [7]

$$
m^{2}=4 \pi T\left[-2+\sum_{i=1}^{24} \sum_{n=1}^{\infty} n\left(N_{n i}+\widetilde{N}_{n i}\right)\right]
$$

with the constraint

$$
\sum_{i=1}^{24} \sum_{n=1}^{\infty} n\left(N_{n i}-\tilde{N}_{n i}\right)=0
$$

Summing (34) over the spectrum (35) exactly reproduces (33). The $\tau_{1}$ integral enforces (36), while $\tau_{2}=s T$.

We have found, not surprisingly, that the free energy of the free string gas reproduces the sum of the free energies of the individual particle states. Now let us consider the cosmological constant. The one loop contribution from a single particle is half the $r=0$ term in (34):

$$
V\left(m^{2}\right)=-\frac{1}{2} \int_{0}^{\infty} \frac{d s}{s}(2 \pi s)^{-d / 2} e^{-m^{2} s / 2} .
$$

Summed over the states (35)-(36), this gives

$$
V_{1 \text { loop }}=-\frac{1}{2} \int_{0}^{\infty} \frac{d s}{s} \int_{-1 / 2}^{1 / 2} d \theta(2 \pi s)^{-13} e^{4 \pi s T}\left|f\left(e^{2 \pi i \theta-2 \pi s T}\right)\right|^{-48} .
$$

Equation (37) has, as $s \rightarrow 0$, the usual ultraviolet divergences. In Eq. (38) these are not cancelled, but in fact are worsened by the sum over an infinite number of bosonic states. This would seem to be a puzzle, since string theories are claimed not to have ultraviolet divergences. Now consider the direct string calculation of the one loop cosmological constant. This is just Eq. (27), with a factor of $-\prod_{\mu} L^{\mu}$ divided out. 
Under the identification $\theta+i s T \rightarrow \tau$, the integrands of the field and string expressions, (38) and (27), are the same, but the regions of integration are different. In the sum over field theories:

$$
-\frac{1}{2}<\tau_{1}<\frac{1}{2}, \quad \tau_{2}>0
$$

In the string theory:

$$
-\frac{1}{2}<\tau_{1}<\frac{1}{2}, \quad \tau_{2}>0, \quad|\tau|>1
$$

The string cosmological constant is different, and ultraviolet finite, since the region $\tau_{2} \rightarrow 0$ is excluded! This seems quite surprising, although the result is actually well known to string experts (see, for example, Rohm [8]). Roughly speaking, what happens is this. The variable $s$ is the proper time for the particle to circle its closed world line. The ultraviolet divergence is from $s \rightarrow 0$. For the string world sheet, however, there is no invariant distinction between this proper time, and the proper distance along which we define the wavefunction of a string state. The discrete group (24) interchanges them. Once the proper time is less than the size of a state, the integration stops, because we would then be double-counting the same surface. We emphasize that this division by the modular group is not an ad hoc procedure: as discussed below Eq. (27), there is an unambiguous normalization to the sum over surfaces. The divergences of the field theory calculation come about because the region (39a) contains, under the modular group, an infinite number of images of (39b). The disagreement between Eqs. (39a) and (39b) is still a puzzle, because string theory might be expected to reduce, in the light cone gauge at least, to a sum of field theories, and we would then expect the full $\frac{1}{2} \omega$ for every state. Again, this puzzle is not unknown in string theory. String theory cannot naively reduce to a sum of field theories because of duality. In a 4-point amplitude $s$ and $t$ exchanges come from the same string surface, and are not to be added; a field theory would overcount by including both. Finally, the only surviving divergence in Eq. (38) is an exponential one as $s \rightarrow \infty$, and its source is clear. It comes from the tachyon term in the sum. Because of the tachyon, long tubular world sheets, whose cross section is the tachyon wave function, are weighted with a positive exponential of their length.

Thus far, we have considered only oriented surfaces, that is, the extended Virasoro-Shapiro model. The partition function of the restricted Virasoro-Shapiro model includes also surfaces with

$$
\begin{aligned}
& x^{\mu}\left(\sigma^{1}, \sigma^{2}+1\right)=x^{\mu}\left(-\sigma^{1}, \sigma^{2}\right)+r \beta \delta^{\mu_{1}}, \\
& x^{\mu}\left(\sigma^{1}+1, \sigma^{2}\right)=x^{\mu}\left(\sigma^{1}, \sigma^{2}\right), \\
& g_{a b}\left(\sigma^{1}, \sigma^{2}+1\right)=g_{a b}\left(-\sigma^{1}, \sigma^{2}\right)(-1)^{a+b}, \\
& g_{a b}\left(\sigma^{1}+1, \sigma^{2}\right)=g_{a b}\left(\sigma^{1}, \sigma^{2}\right) .
\end{aligned}
$$

This can be regarded as the insertion of the twist operator $\Omega: \sigma^{1} \rightarrow-\sigma^{1}$, into the oriented path integral. There is an additional factor of 2 in $\operatorname{order}(\widetilde{D})$ from the orientation-changing transformation

$$
\sigma_{1} \rightarrow-\sigma_{1} \sigma_{2} \rightarrow \sigma_{2}
$$

so that $F_{R V S M}$ contains a factor of $\frac{1}{2}(1+\Omega)$ as compared to $F_{E V S M}$ : this precisely excludes the $\Omega=-1$ states. 
As a final check, consider the one-loop amplitude for the scattering of $M$ tachyons of momenta $p_{1}, \ldots, p_{M}[2,7]$. Insert

$$
\prod_{i=1}^{M} \kappa_{0} \int d^{2} \sigma_{i} \sqrt{g\left(\sigma_{i}\right)} e^{i p_{i} \cdot x\left(\sigma_{i}\right)}
$$

into the functional integral. The integration over $\bar{x}^{\mu}$ gives, as $L^{\mu} \rightarrow \infty,(2 \pi)^{d} \delta\left(\sum_{i} p_{i}\right)$. Then,

$$
\begin{aligned}
A\left(p_{1}, \ldots, p_{M}\right)= & (2 \pi)^{26} \delta\left(\sum_{i} p_{i}\right) \prod_{i=1}^{M}\left(\tau_{2} \kappa_{0} \int d^{2} \sigma_{i}\right) \\
& \cdot \int_{F} \frac{d^{2} \tau}{4 \pi \tau_{2}{ }^{2}} e^{4 \pi \tau_{2}}\left(2 \pi \tau_{2}\right)^{-12}\left|f\left(e^{2 \pi i \tau}\right)\right|^{-48} \exp -\frac{1}{2} \sum_{j, k} p^{\mu}{ }_{j} p^{\nu}{ }_{k}\left\langle x^{\mu^{\prime}}\left(\sigma_{j}\right) x^{\nu^{\prime}}\left(\sigma_{k}\right)\right\rangle .
\end{aligned}
$$

The term $j=k$ is divergent; this is absorbed in a redefinition of $\kappa_{0}$. Note that it is not correct merely to normal order (exclude $j=k$ ), because the constant absorbed into $\kappa_{0}$ would then be $\tau$ and topology dependent. Rather define the $j=k$ term by $\zeta$ function regularization. The double sum in the propagator can be evaluated as in the Appendix to give

$$
\left\langle x^{\prime}\left(\sigma_{j}\right) x^{\prime}\left(\sigma_{k}\right)\right\rangle=\frac{\tau_{2}\left(\sigma^{2}{ }_{j}-\sigma_{k}^{2}\right)^{2}}{2 T}+\frac{3 \tau_{2}}{4 T}-\frac{1}{2 \pi T} \ln \left|\frac{\theta_{1}\left(v_{j k} \mid \tau\right)}{f\left(e^{2 \pi i \tau}\right) e^{i \pi \tau / 4}}\right|, \quad j \neq k,
$$

where $v_{j k}=\sigma^{1}{ }_{j}+\tau \sigma^{2}{ }_{j}-\sigma^{1}{ }_{k}-\tau \sigma^{2}{ }_{k}$ and the Jacobi $\theta$-function is discussed in [7]. At $j=k$,

$$
\left\langle x^{\prime}\left(\sigma_{j}\right) x^{\prime}\left(\sigma_{j}\right)\right\rangle=\frac{3 \tau_{2}}{4 T}+\frac{1}{4 \pi T t}+\frac{1}{2 \pi T} \ln \left(\frac{e^{\gamma}}{4 \pi}\right)-\frac{1}{2 \pi T} \ln \left|\frac{\theta_{1}^{\prime}(0 \mid \tau)}{2 \pi f\left(e^{2 \pi i \tau}\right) e^{i \pi \tau / 4}}\right|,
$$

where $t \rightarrow 0$ at the $\zeta$-function regulator is removed. Using $\sum_{i} p_{i}=0, p_{i}{ }^{2}=8 \pi T$, and defining a renormalized $\kappa_{R}$ to agree with [7],

$$
\kappa_{R}=8 \pi \lim _{t \rightarrow 0} \kappa_{0}(t) e^{-2 \gamma-1 / t}
$$

Equation (39) becomes

$$
\begin{aligned}
A\left(p_{1}, \ldots, p_{M}\right)= & (2 \pi)^{26} \delta\left(\sum_{i} p_{i}\right) T^{13} \prod_{i=1}^{M}\left(2 \pi \kappa_{R} \tau \int d^{2} \sigma_{i}\right) \\
& \cdot \int_{F} \frac{d^{2} \tau}{4 \pi \tau_{2}{ }^{2}} e^{4 \pi \tau_{2}}\left(2 \pi \tau_{2}\right)^{-12}\left|f\left(e^{2 \pi i \tau}\right)\right|^{-48} \prod_{j>k} \chi\left(v_{j k}, \tau\right)^{p_{j} \cdot p_{k} / 2 \pi T},
\end{aligned}
$$

where

$$
\chi(v, \tau)=2 \pi \exp \left\{\frac{-\pi(\operatorname{Im} v)^{2}}{\tau_{2}}\right\}\left|\frac{\theta_{1}(v \mid \tau)}{\theta_{1}^{\prime}(0 \mid \tau)}\right|
$$

This agrees with the expression found by operator methods, given in refs. [2,7] 
for $T=1 / \pi$ (except that the factor of $1 / 2$ from $\operatorname{order}(\widetilde{D})$ was omitted in [2] and in Eq. 6.3 of [7]; Eq. 6.13 of [7] differs by an additional factor of 2.)

In summary, the path integral of Polyakov is well defined for $L=1, d=26$. The keys which make it unambiguous are Weyl invariance, which fixes $\mu^{2}$ and the locality of the measure, Eq. (28). It is also a nice exercise to apply the methods used here to the reparameterization invariant path integral for the relativistic scalar propagator. Our observations about the calculations of the cosmological constant come as a surprise to the field theorist, but are in agreement with string lore.

\section{Appendix}

We wish to evaluate

$$
\ln \operatorname{det}^{\prime}=\sum_{n_{1}, n_{2}}^{\prime} \ln \left[\frac{4 \pi^{2}}{\tau_{2}{ }^{2}}\left(n_{2}-n_{1} \tau\right)\left(n_{2}-n_{1} \bar{\tau}\right)\right],
$$

where the prime omits $n_{1}=n_{2}=0$. The finite part of the $\mu^{2}$ counterterm is fixed by the requirement that the $x$-integral be invariant under $g_{a b} \rightarrow e^{\lambda} g_{a b}$. $\zeta$-function regularization, since it has no scale, gives the right finite part without additional subtraction. Thus, define

$$
\begin{aligned}
\ln \operatorname{det}^{\prime} & =-\lim _{s \rightarrow 0} \frac{d}{d s} \sum_{n_{1} n_{2}}\left[\frac{4 \pi^{2}}{\tau_{2}^{2}}\left(n_{2}-n_{1} \tau\right)\left(n_{2}-n_{1} \bar{\tau}\right)\right]^{-s} \\
& =-\lim _{\substack{s \rightarrow 0 \\
m^{2} \rightarrow 0}}\left\{\frac{d}{d s}\left(\frac{4 \pi^{2}}{\tau_{2}^{2}}\right)^{-s} \sum_{n_{1} n_{2}}\left[\left(n_{2}-n_{1} \tau\right)\left(n_{2}-n_{1} \bar{\tau}\right)+m^{2}\right]^{-s}-\left(4 \pi^{2} m^{2} / \tau_{2}{ }^{2}\right)^{-2}\right\} .
\end{aligned}
$$

The $n_{2}$ sum is converted to a integral using the Sommerfeld-Watson transformation:

$$
\begin{aligned}
\ln \operatorname{det}^{\prime}= & \lim _{\substack{s \rightarrow 0 \\
m^{2} \rightarrow 0}}-2 \frac{d}{d s}\left[\left(\frac{4 \pi^{2}}{\tau_{2}{ }^{2}}\right)^{-s} \int_{c} d z \sum_{n_{1}} \frac{e^{i \pi z}}{2 i \sin \pi z}\left[\left(z-n_{1} \tau_{1}\right)^{2}+n^{2}{ }_{1} \tau_{2}{ }^{2}+m^{2}\right]^{-s}+\text { h.c. }\right] \\
& +\frac{d}{d s}\left[\left(\frac{4 \pi^{2}}{\tau_{2}{ }^{2}}\right)^{-s} \int_{c} d z \sum_{n_{1}}\left[\left(z-n_{1} \tau_{1}\right)^{2}+n_{1}{ }^{2} \tau_{2}{ }^{2}+m^{2}\right]^{-s}+\text { h.c. }\right] \\
& -\left(\frac{4 \pi^{2} m^{2}}{\tau_{2}{ }^{2}}\right)^{-s} \ln \left(\frac{4 \pi^{2} m^{2}}{\tau_{2}{ }^{2}}\right) .
\end{aligned}
$$

The contour passes above the real axis, from $+\infty+i \varepsilon$ to $-\infty+i \varepsilon$. The first term in brackets converges at $s=0$ and gives

$$
4 \sum_{n_{1}=1}^{\infty} \ln \left|1-e^{2 \pi i n_{1} \tau}\right|+2 \ln (2 \pi m)+O\left(m^{2}\right)
$$


The second converges for $s>1$ :

$$
\begin{aligned}
\frac{d}{d s}[ & \left.\frac{2^{1-2 s} \sin \pi s}{\cos \pi s} \frac{\Gamma^{2}(1-s)}{\Gamma(2-2 s)}\left(m^{1-2 s}+2 \tau_{2}{ }^{1-2 s} \zeta(2 s-1)+O\left(m^{2}\right)\right)\right] \\
& \underset{\substack{s \rightarrow 0 \\
m^{2} \rightarrow 0}}{\rightarrow} 4 \pi \tau_{2} \eta(-1)=-\frac{\pi}{3} \tau_{2} .
\end{aligned}
$$

Equation (22) follows. The term (A.5) comes from the zero point energies of the string oscillators, and produces the exponential term in Eq. (27), (33). Thus (cf. (34))

$$
m_{\text {tachyon }}^{2}=4 \pi T(d-2) \zeta(-1)
$$

as in ref. [9].

Acknowledgements. I have benefited from discussions with P. Candelas, E. Martinec, L. Mezincescu, G. Moore, P. Nelson, W. Thurston, R. Woodard, and particularly W. Fischler and S. Weinberg. I am grateful to J. Govaerts, S. Weinberg, and G. Gilbert for comments on the manuscript. Supported in part by Robert A. Welch Foundation and NSF 8304629.

\section{References}

1. Polyakov, A. M.: Quantum geometry of bosonic strings. Phys. Lett. 103B, 207 (1981)

2. Shapiro, J. A.: Loop graph in the dual-tube model. Phys. Rev. D5, 1945 (1972)

3. Friedan, D.: In: 1982 Les Houches Summer School. Zuber, J.-B., Stora, R. (eds.) Amsterdam: North Holland 1984

4. Alvarez, O.: Theory of strings with boundaries: Fluctuations, topology and quantum geometry. Nucl. Phys. B216 125 (1983)

5. Schiffer, M., Spencer, D. C.: Functionals of finite Riemann surfaces Princeton NJ: Princeton University Press 1954

6. Weinberg, S.: Texas preprint UTTG-05-85 (1985)

7. Schwarz, J. H.: Superstring theory. Phys. Rep. 89, 223 (1982)

8. Rohm, R.: Spontaneous supersymmetry breaking in supersymmetric string theories. Nucl. Phys. B237, 553 (1984)

9. Brink, L., Nielsen, M. B.: A simple physical interpretation of the critical dimension of space-time in dual models. Phys. Lett. 45B, 332 (1973)

Communicated by A. Jaffe

Received June 24, 1985

Note added in proof: To define $\left\langle x^{\prime}(\sigma) x^{\prime}(\sigma)\right\rangle$, the definition $\left\langle x^{\prime}(\sigma) x^{\prime}(\sigma)\right\rangle_{R}=\lim \left\langle x^{\prime}(\sigma) x^{\prime}\left(\sigma^{\prime}\right)\right\rangle+$ $(1 / 4 \pi) \ln d\left(\sigma, \sigma^{\prime}\right)^{2}$, where $d\left(\sigma, \sigma^{\prime}\right)$ is the invariant distance, is much simpler in practice than the $\xi$-function. I am grateful to $S$. Weinberg and $E$. D'Hoker for discussions of this. 
